\title{
水稻粒形性状的遗传及相关基因定位与克隆研究进展
}

高志强，占小登，梁永书，程式华，曹立勇

中国水稻研究所，水稻生物学国家重点实验室/国家水稻改良中心，杭州 310006

摘要: 作物育种的首要目标是提高产量。水稻粒形是与水稻产量性状直接相关, 与品质性状存在着密切关系的 数量性状, 其评价指标主要是粒长、粒宽、粒厚、长/宽和长/厚。近年来, 水稻粒形的数量遗传研究取得了重要 进展, 并成功定位克隆了一批控制水稻粒形的基因。文章综述了水稻粒形的经典遗传研究、QTL 定位、粒形基 因的克隆和功能分析以及在水稻超高产育种中的利用。

关键词: 水稻; 粒形; QTL 定位; 基因功能

\section{Progress on genetics of rice grain shape trait and its related gene mapping and cloning}

\author{
GAO Zhi-Qiang, ZHAN Xiao-Deng, LIANG Yong-Shu, CHENG Shi-Hua, CAO Li-Yong
}

State Key Laboratory of Rice Biology/China National Center for Rice Improvement, China National Rice Research Institute, Hangzhou 310006, China

\begin{abstract}
Increase of crop production is the primary goal of crop breeding. Rice grain shape is a quantitative trait that is directly related to yield traits and has a close relationship with quality traits. The evaluation of grain shape is mainly grain length, grain width, grain thickness, length/width, and length/thickness. In recent years, the quantitative genetic research on rice grain shape has made a significant progress and a number of important genes associated with rice grain shape have been cloned. This paper reviews the classic genetic analysis on rice grain traits, QTL mapping, grain shape gene cloning and functional analysis, and their application in rice breeding for super high yield.
\end{abstract}

Keywords: rice; grain shape; QTL mapping; gene function

水稻是重要的粮食作物, 世界上有 100 多个国 家种植水稻 ${ }^{[1]}$, 世界 $50 \%$ 以上的人口以水稻为主食 ${ }^{[2]}$, 水稻提供了世界人口约 $21 \%$ 、东南亚国家约 $76 \%$ 的 能量摄入需求 ${ }^{[3]}$ 。中国是一个水稻生产和消费大国, 据FAO(Food and Agriculture Organization of the United Nations)在 2003 至 2005 年间对中国饮食结构
的统计, 中国人的谷物摄取量为每人每天 436 克, 其中水稻占 49.5\%(http://faostat.fao.org/)。到 2030 年, 中国人口将达到 16 亿, 粮食需求总量将从 2010 年 的 5.8 亿吨增至 7.4 亿吨才能满足需要 ${ }^{[4]}$, 这说明水 稻对保障我国粮食安全具有重要作用。20 世纪 50 年代以来, 通过矮化育种和杂种优势利用, 实现了

\footnotetext{
收稿日期: 2010-06-11; 修回日期: 2010-07-13 30623006)资助

作者简介: 高志强, 在读硕士研究生, 专业方向 : 水稻遗传育种。E-mail: yzxjgzq@yahoo.cn

通讯作者: 曹立勇, 博士, 研究员, 研究方向 : 水稻遗传育种。E-mail: caolycgf@mail.hz.zj.cn

出版时间:2011-2-17 14:52

IRL: http://www.cnki.net/kcms/detail/11.1913.R.20110217.1452.000.html
}

基金项目: 抗病转基因水稻新品种培育(编号 : 2008ZX08001-002), 国家 948 计划资助项目(编号：2006-G51)和国家自然科学基金专项(编号 : 
水稻产量的两次飞跃 ${ }^{[5,6]}$, 但近 20 年来, 水稻单产 徘徊不前 ${ }^{[7]}$, 如何在水稻种植面积基本保持稳定或 略有下降的情况下增加水稻产量, 保障我国粮食安 全, 是当前面临的一个新课题。水稻粒形性状是与 产量性状直接相关的重要农艺性状之一，阐明水稻 粒形的遗传与发育机理并将之应用于育种是提高水 稻单产的一个重要手段 ${ }^{[8]}$ 。同时，由于粒形性状与水 稻的外观品质、加工品质、蒸煮和食味品质等都存 在着密切的关系 ${ }^{[9]}$, 因而, 水稻的粒形性状不仅影 响稻谷产量, 而且影响稻米的品质, 并对水稻的产 量形成及品质形成都具有重要作用。本文从水稻粒 形性状的经典遗传、重要粒形相关性状的QTL定位 和基因克隆等方面，综述了前人在水稻粒形相关性 状方面的研究成果，以期为水稻粒形相关基因在水 稻超高产育种中的利用提供指导。

\section{1 水稻粒形相关性状的经典遗传研究进展}

水稻的粒形是水稻重要的产量和品质相关性状, 水稻颖花中的子房壁发育成颖果的果皮，胚珠中的 珠被珠心发育成种皮。胚囊中两个极核与花粉中的 精核通过双受精发育成三倍体胚乳、珠孔口处的卵 核与花粉中的另一精核通过单受精发育成二倍体胚, 但成熟颖果(谷粒)的颖壳、果皮和种皮则是来自二倍 体的母体植株。由于二倍体胚、三倍体胚乳均为子 代组织, 其营养需要由二倍体母体植株提供, 所以 成熟谷粒的外观品质性状会同时受到胚乳、母体植 株和细胞质等不同遗传体系的控制 ${ }^{[10]}$ 。

\section{1 水稻粒形性状的遗传与相关性研究}

水稻的粒形性状主要包括粒长、粒宽、粒厚、 长/宽和长/厚等 ${ }^{[11]}$ 。许多实验表明粒长的遗传是单基 因、双基因、多基因或微效多基因控制，但国内外 的学者更多认为粒长以多基因控制为主 ${ }^{[12]}$, 并兼有 不完全显性作用，其显性作用因品种而异，一般认 为显性作用长 $>$ 中 $>$ 短 $>$ 极短, 但也存在短粒显性 和长粒隐性 $[13]$, 例如 $M i 3(t)$ 基因控制水稻籽粒长度, 小粒对大粒完全显性 [14]。粒宽和粒厚多为正态分布, 说明该性状受多基因控制，但也有受主效基因或单 基因控制的，如 $G W 5^{[15]}$ 和 $q S W 5^{[16]}$ ，而且粒宽和粒厚 的显性方向因组合而异，郭益全等 ${ }^{[17]}$ 经回归分析和 亲本显性次序分析后认为粒长和长/宽为单方向显
性，而粒宽则表现为双方向显性。粒重是构成产量 性状的重要因素之一, 它是粒长、粒宽和粒厚的综

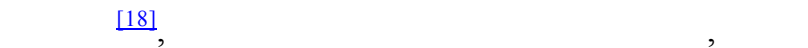
呈正态分布 ${ }^{[19]}$ 。所以谷粒的粒长、粒宽、粒厚、长/ 宽和粒重都属于多基因控制的数量性状 ${ }^{[20]}$ ，同时不 同性状间存在着相关性。莫惠栋 ${ }^{[13]}$ 认为粒宽和粒厚 与粒重成正相关，长宽比与粒重呈负相关，粒长与 粒重之间既可正相关，亦可表现负相关。石春海等 ${ }^{[21]}$ 研究了粒形及产量性状的加性相关和显性相关, 结 果发现农艺性状间的加性相关比显性相关重要, 粒 形性状间的遗传相关以加性相关为主，只有粒宽和 长宽比性状间同时存在显著的加性相关和显性相关， 而且多数粒形性状与每穗粒数、结实率、粒重的加 性相关要大于其他性状。

\section{2 水稻粒形性状的遗传效应研究}

在对控制水稻粒形性状的各种遗传效应的分析 中发现, 䊁米长、长/宽和长/厚等性状以母体遗传率 为主, 䊁米宽和䊁米厚则以胚乳直接遗传率为主 ${ }^{[21]}$ 。 祈祖白等 ${ }^{[22]}$ 利用籼稻品种研究籽粒外观品质, 认为 米粒的形态特征可能是由植株的基因控制，细胞质 基因的作用很小; 而易小平等 ${ }^{[23]}$ 利用 6 个同核异质 雄性不育系研究发现稻米外观品质等性状均受到不 育系细胞质类型的影响。水稻粒形狭义遗传率最高 的是粒长 $(95.3 \% \sim 97.5 \%)$, 其次是粒宽(约 $94.5 \%)$, 最低是粒厚 $(78.3 \%)^{[13]}$ 。对于粒形性状的加性和显性 效应, 符福鸿等 ${ }^{[24]}$ 的研究结果认为谷粒长、宽、长/ 宽和千粒重等 4 个性状均为加性基因效应起主导作 用。石春海等 ${ }^{[25]}$ 对控制籼稻稻米形状表现的环境互 作效应进行了分析，结果发现粘米长、糙米长/宽和 糙米长/厚主要受制于遗传主效应，糙米宽和䊁米厚 的表现则主要受到环境互作效应的影响, 所以粒长 的表现更多的是受遗传控制，环境影响很小，而粒 宽和粒厚的表形存在明显的基因型与环境互作效应, 与灌浆充实期的环境条件关系密切。

以上有关水稻谷粒外观品质性状的遗传效应研 究都是基于成熟的谷粒，并不能反映粒形性状在不 同发育阶段或时间段内净遗传效应的大小和变化, 而这些遗传效应分析是研究基因的时空表达调控所 需要的, 朱军 ${ }^{[26]}$ 提出了可以估算数量性状在不同发 育时期的条件遗传方差分量和净遗传效应值的遗传 
模型和统计分析方法。石春海等 ${ }^{[27]}$ 对籼稻稻米的粒 长和粒宽发育遗传进行了深入研究, 非条件的遗传 方差分析表明水稻的䊁米长和粘米宽性状在不同灌 浆期会同时受到三倍体胚乳核基因、细胞质基因和 二倍体母体植株核基因控制，而且在不同灌浆期控 制粒长的母体遗传效应能占遗传总效应的 $50 \%$ 以上， 而细胞质遗传效应只占到遗传总效应的 $10 \%$ 左右, 说明母体效应是控制粒长的主效应，而细胞质效应 对粘米粒长的影响也比较重要, 并且基因的加性方 差占总遗传方差的 $70.2 \% \sim 77.8 \%$, 这些结果和前面 的粒形遗传分析结果是一致的。对粗米粒长性状的 条件方差分析结果表明在水稻不同灌浆期的胚乳核 基因、细胞质基因和母体植株核基因都有新的遗传 效应表达, 在开花后 8 14 天灌浆期时基因新表达产 生的净遗传效应对控制粒长很重要, 而开花后 8 14 天或 15 21 天这两个时期中三倍体胚乳核基因新表 达的遗传效应要比母体植株核基因的表达效应对粒 形发育影响更重要。䊁米粒宽性状的非条件遗传方 差分析表明, 虽然开花后 7 天䊁米宽度主要受制于 胚乳遗传效应 $(42.56 \%)$, 在第 14、21、28 天时的母 体遗传效应 $(45.57 \%$ 71.51\%)则要大于其他遗传效 应, 而在整个灌浆期, 细胞质效应对糙米宽的影响 (18.46\% 29.01\%)要比细胞质对粘米长的影响更重 要。石春海等 ${ }^{[28]}$ 对籼稻 4 个发育时期的精米重量性 状也进行了发育遗传研究, 研究结果再次验证了三 倍体胚乳、二倍体母体植株和细胞质效应对精米重 量的控制作用, 而且胚乳、细胞质和母体植株中控 制精米重量性状表现的基因以稻米发育前期表达为 主, 其中开花后第 8 14 天是控制精米重量性状的基 因表达最为活跃的时期。这说明控制稻米粒形的基 因和控制稻米粒重的基因在稻米发育早期的表达很
重要, 它们可能属于不同基因对稻米发育进行控制, 或属于同一基因而具有一因多效，同时影响粒形和 粒重。

\section{2 水稻粒形相关性状 QTL 的定位}

经典遗传分析结果表明水稻的许多重要农艺性 状属于数量性状, 例如产量性状、品质性状、对病 虫害的水平抗性和对不良环境因子的耐性等 [29 31]。 对于数量性状的研究必须用有别于质量性状的研究 方法, 将控制数量性状的位点分解成单个QTL(基因) 进行研究, 在构建遗传分离群体和遗传图谱的基础 上进行QTL初定位, 再用高代回交群体或NIL-QTL 开展精细定位 ${ }^{[32,33]}$ 。1994 年首次发表水稻产量性状 的QTL定位研究结果 ${ }^{[34]}$, 至今已经定位到上千的水 稻数量性状位点, 根据Gramene QTL (http://www. gramene.org/qt1/)收录的从 1994 年到 2006 年 6 月的 水稻QTL定位信息, 整理分析了有关水稻粒形定位 结果。在Gramene的 QTL定位结果数据库中共收录 栽培稻QTL定位数据 8646 条, 其中产量相关性状的 QTL最多, 为 2060 个; 控制水稻粒形性状的QTL为 219 个, 已经定位到的控制水稻粒形相关性状QTLs 见表 1。根据Gramene QTL提供的粒形QTL信息中的 作图群体信息发现，水稻粒形QTL定位结果主要来 自 Zhan97/Ming63 ${ }^{[35,36]} 、$ Asominori/IR24 ${ }^{[37]}$ V20A/glab ${ }^{[38]}$ 、Nipponbare/Kasalath (http://rgp.dna.affrc. go.jp/)、Rei/Yamd ${ }^{[39]} 、$ Caiapo/IRGC103544 $4^{[40]} 、$ IR64/ $\mathrm{Azu}^{[41]} 、 \mathrm{Lab} / \mathrm{Bla}^{[42]} 、 \mathrm{~W} 1944 / \mathrm{Peik}{ }^{[43]}$, 而有关水稻粒形 的长、宽QTL定位结果主要来自前面 4 个群体, 例如 GS3 供体亲本是明恢 63、GW2 供体亲本是WY3、 GW5 供体亲本是Asominori、qSW5 的供体亲本是 Kasalath (http://www.ricedata.cn/gene/gene_tgw.htm)。

表 1 水稻不同粒形性状 QTL 定位的染色体分布表

\begin{tabular}{|c|c|c|c|c|c|c|c|c|c|c|c|c|}
\hline 粒形性状 & Chr.1 & Chr.2 & Chr. 3 & Chr.4 & Chr.5 & Chr.6 & Chr.7 & Chr.8 & Chr.9 & Chr.10 & Chr.11 & Chr.12 \\
\hline 种子粒长 & 4 & 1 & 9 & 3 & 0 & 2 & 1 & 0 & 2 & 2 & 2 & 0 \\
\hline 种子粒宽 & 4 & 3 & 6 & 1 & 5 & 3 & 1 & 2 & 1 & 2 & 3 & 0 \\
\hline 种子粒厚 & 0 & 1 & 0 & 1 & 1 & 0 & 0 & 0 & 0 & 0 & 0 & 0 \\
\hline 种子粒形 & 0 & 0 & 2 & 0 & 2 & 0 & 0 & 0 & 0 & 0 & 0 & 0 \\
\hline 种子 L/W & 1 & 1 & 4 & 1 & 0 & 1 & 1 & 0 & 0 & 0 & 0 & 0 \\
\hline 糙米粒长 & 9 & 9 & 15 & 5 & 2 & 4 & 2 & 0 & 0 & 1 & 1 & 1 \\
\hline 糙米粒宽 & 4 & 2 & 1 & 0 & 17 & 4 & 1 & 1 & 0 & 1 & 3 & 1 \\
\hline 糙米重 & 3 & 0 & 4 & 0 & 5 & 1 & 3 & 1 & 1 & 0 & 4 & 0 \\
\hline 糙米 $\mathrm{L} / \mathrm{W}$ & 4 & 1 & 14 & 1 & 16 & 1 & 0 & 0 & 0 & 0 & 0 & 3 \\
\hline
\end{tabular}


注: Chr.: chromosome ; L/W: length/width。

从表 1 可见，在第 1 、第 2 、第 3 及第 5 染色体 上定位到的水稻粒形QTL数较多, 分别为 $29 、 18$ 、 55 和 48 个, 其中第 3 染色体定位到的控制粒长和长/ 宽的QTL数较多, 第 5 染色体定位到控制粒宽和长/ 宽的QTL数较多。根据定位结果推测, 第 3 染色体和 第 5 染色体可能是水稻粒形相关基因分布的热点区 域，目前已在这两条染色体上成功克隆了控制水稻 粒重的基因 $G S 3^{[44]} 、 G W 5^{[15]}$ 和 $q S W 5^{[16]}$ 。另还可见, 9 个粒形性状中, 粒长、粒宽性状定位结果最多, 而有 关粒厚、粒形定位结果很少, 由于粒长和粒宽是长/ 宽的相关性状，所以长/宽性状的定位也是可以的。

水稻粒形性状的QTL定位结果表明，同一性状 能定位到不同的染色体上(表 1)，而在同一染色体同 一区域也可能定位到不同粒形性状的QTL。例如在 第 5 染色体, Asominori/IR24 [37]群体的 8.4 31.4 cM区 间，Nipponbare/Kasalath (RGP 2000)群体的 12 19

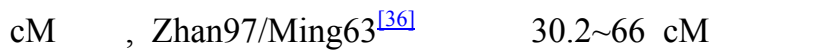
分别存在粒宽和长/宽效应位点, Nipponbare/Kasalath (RGP 2000)群体在 70.5 74.2 cM区间存在谷粒长、谷 粒长/宽的效应位点，而Zhan97/Ming63 ${ }^{[35]}$ 群体在 16.1 49.2 cM区间存在粒宽、粒形、谷粒宽、谷粒长/ 宽和谷粒重的效应位点; 在第 3 染色体, $\mathrm{V} 20 \mathrm{~A} / \mathrm{glab}^{[38]}$ 群体的 31.2 76.9 cM区间, Asominori/IR24 ${ }^{[37]}$ 群体的 63.4 81.5 cM区间, IR64/Azu ${ }^{[411}$ 群体在 $141.4 \mathrm{cM}$ 位置, 都分别存在谷粒长、谷粒长/宽的效应位点; Nipponbare/Kasalath (RGP 2000)群体在 85.2 87.4 cM区间, W1944/Peik ${ }^{[43]}$ 群体在 $126 \mathrm{cM}$ 位置, 分别都存在谷粒 宽、谷粒长/宽的效应位点, 而Zhan97/Ming63 $3^{[35]}$ 群体 在 77.7 88.1 cM区间同时存在谷粒长、谷粒长/宽、

粒长、粒形的效应位点。
QTL定位频率较高的染色体或染色体区域，往 往反应出在不同的遗传背景和条件下不同性状的强 表达，而对于某性状的QTL定位，往往在高频区域 (QTL热点区域)定位到该性状 QTL的可能性大 ${ }^{[45]}$, 例如第 3 染色体的着丝粒附近, 已经有很多粒形相 关QTL的定位结果。水稻的全基因组测序分析结果 也表明，基因在染色体上并非随机排列，而是存在

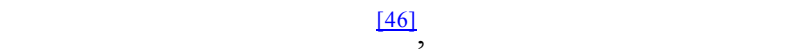
状QTL簇的存在形式以及某一基因的一因多效机理 有深入了解, 这对于分子标记辅助选择或多性状的 同步改良将提供很好的途径，特别是能打破不利农 艺性状的连锁, 例如籼稻粒形改良, 增加粒长粒厚 而减少粒宽，从而在提高粒重的同时能减少对稻米 品质的影响。

\section{3 水稻重要粒形基因的克隆和功能分析}

水稻重要产量相关性状基因的定位、克隆和功 能分析, 有助于水稻产量性状的分子遗传改良, 提 高水稻单产。2006 2008 年, 国内外科学家纷纷克隆 了控制水稻粒形的基因 $G S 3^{[44]} 、 G W 2^{[47]} 、 G W 5^{[15]}$ 、 $q S W 5^{[16]}$, 同时也定位克隆出许多重要的产量性状相 关基因(表 2)。

水稻重要粒形基因的克隆主要还是采用图位克 隆的方法, 即通过构建暂时性群体 $\left(\mathrm{F}_{2}\right)$ 或永久性群 体(RIL)，在绘制遗传图谱的基础上进行粒形性状的 QTL初定位，发现具有较大效应值的QTL，并针对 目标区间构建高代回交群体, 并从中篮选背景为显

表 2 已经克隆的水稻重要粒形基因和部分产量相关基因

\begin{tabular}{|c|c|c|c|}
\hline 基因符号 & 基因中文名称(或性状功能) & 染色体 & 文献 \\
\hline GW5 & 粒宽和粒重主效控制基因 & 5 & {$[15]$} \\
\hline qSW5 & 粒宽基因 & 5 & {$[16]$} \\
\hline GS3 & 粒长粒重主效控制基因 & 3 & [44] \\
\hline GW2 & 粒宽和粒重主效控制基因 & 2 & [47] \\
\hline GIF1 & 籽粒充实度基因 & 4 & {$[48]$} \\
\hline Ghd7 & 每穗实粒数、株高和抽穗期多效控制基因 & 7 & [49] \\
\hline Gn1a & 每穗实粒数基因 & 1 & [50] \\
\hline$D E P 1$ & 直立型密穗基因 & 9 & {$[51]$} \\
\hline IPA1 & 理想株型 (少分葟亲, 抗倒伏, 增加谷粒产量) & 8 & {$[52]$} \\
\hline
\end{tabular}


WFP 增加稻穗一次枝梗数和谷粒产量 性亲本而在目标区间为杂合的单株通过自交成为近 等基因系; 再通过QTL方法将目标区间的遗传距离 缩减到更小 ${ }^{[32]}$, 这可以防止由于遗传背景的不纯对 目标区间QTL定位的影响; 最后发展新的标记和选 取极端表型的隐性单株进行重组交换单株的篮选, 将目标基因定位的距离缩小到 $100 \mathrm{~kb}$ 以内。

$G S 3^{[44]}$ 是一个控制粒长和粒重的主效基因，在 利用珍汕 97/明恢 63 的 $\mathrm{F}_{2: 3}$ 和RIL群体进行水稻产量 性状的QTL定位研究时, 发现在第 3 染色体上存在 一个控制粒形性状，并且效应较大的QTL ${ }^{[35,54]}$ 。Fan 等 ${ }^{[44]}$ 通过构建高代回交群体 $\mathrm{BC}_{3} \mathrm{~F}_{1}$, 并从中挑选目 标区间杂合，而背景为明恢 63 的单株发展 $\mathrm{BC}_{3} \mathrm{~F}_{2}$ (GS3-NIL)群体进行该QTL的精细定位, 并从中篮选 隐性表型单株进行重组筛选, 使GS3 定位在 $7.9 \mathrm{~kb}$ 范围。GS3 基因编码 232 个氨基酸, 对GS3 的蛋白序 列进行预测和分析, 发现其具有 4 个已知功能的区 域和结构域, 其中最重要的结构域是第 97 117 位氨 基酸构成的跨膜区，以及 C端类似于VWFC模体的半 胱氨酸富集区。GS3 基因导致粒长变化的突变位点是 在第二个外显子中发生 C-A单碱基突变(SNP),使半胱 氨酸密码子(TGC)变成终止密码子(TGA), 导致在长 粒品种中发生GS3 翻译蛋白C端 178 个氨基酸的断 开而提前翻译终止。根据GS3 的表达时期和表达组 织, 推测GS3 通过控制胚珠的发育来调控种子大小 ${ }^{[55,56]}$, 而且 Takano-Kai等 ${ }^{[55]}$ 研究发现GS3 的突变型 和野生型在mRNA转录水平上是相似的, 所以 GS3 对粒形的影响在于蛋白翻译水平, 即蛋白功能的失 活, 其失活原因主要在于GS3 编码蛋白的VWFC模 体，因为GS3 编码跨膜蛋白，并含有VWFC模体，其 翻译过程中由于产生终止密码子的无义突变, 导致多 肽链合成的提前终止, 而这种提前终止造成功能蛋白 的VWFC模体的缺失, 而这种VWFC模体缺失导致膜 蛋白功能失活, 因为有研究证明VWFC结构域在蛋白 与蛋白的相互作用过程中起到重要的作用 ${ }^{[57,58]}$, 例如 VWFC模体接合 TGF- $\beta$ 家族成员 ${ }^{[59]}$ ，所以推测VWFC 的功能在于通过扰乱TGF- $\beta$ 超家族成员的受体位点来 调节生长因子的信号通路 [60]。

$G W 2^{[47]}$ 是一个控制粒宽和粒重的主效基因, Song等 ${ }^{[47]}$ 利用WY3/FAZ1 杂交产生的 $\mathrm{F}_{2}$ 分离后代进 行了QTL初定位, 主要利用了高代回交群体和篮选
高代回交分离群体中的隐性单株, GW2 被定位在 8.2 $\mathrm{kb}$ 范围。Song等 ${ }^{[47]}$ 研究发现 $G W 2$ 基因编码蛋白是含 有 425 个氨基酸残基的多肽, 含有与锌指蛋白相似 的蛋白序列，而且在第四外显子中发生单碱基的缺 失, 产生转录终止密码, 导致 310 个蛋白残基的断开, 只能转录翻译出含有 115 个残基的多肽。GW2 在水稻 苗的茎、根、花序分生组织、幼穗、叶和颖花以及 受精 4 天的胚乳中组织性表达, 这不同于GS3 的组 织特异性表达, 并且 GW2 mRNA的表达在FAZ1 和 $\mathrm{NIL}(G W 2)$ 间没有差异, 即转录水平上和 $G S 3$ 又是相 同的, 所以 $G W 2$ 和GS3 对粒形的影响在于功能蛋白 的无义突变造成蛋白功能层面上的差异。用受精前 的颖花和成熟谷粒进行切片观察发现NIL(GW2)的 宽度增加在于颖花内外稃组织细胞数量的增加, 而 非细胞大小的变化, 说明 $G W 2$ 可能参与了细胞分化, 而NIL(GW2)胚乳大小差异在于细胞大小差异, 而非 细胞数量, 说明 $G W 2$ 可能也参与了细胞伸长 ${ }^{[47]}$ 。

qSW5、GW5 是控制粒宽的主效基因, Shomra等 ${ }^{[16]}$ 利用Nipponbare/Kasalath杂交自交产生的 $F_{2}$ 分离后 代进行QTL初定位时发现 $q S W 5$ 位点, 而 $G W 5^{[15]}$ 的 初定位用到了CSSL和RIL群体，其好处是可以对定 位到的结果进行多年多点分析，从而找到效应值很 大又同时稳定(不受环境影响)可靠的QTL, GW5 被 定位在 $21 \mathrm{~kb}$ 范围, $q S W 5$ 被定位在 $2.2 \mathrm{~kb}$ 范围。虽然 这两个基因由不同的研究人员利用不同的供体亲本 (Kasalath和IR24)定位，但两基因实属同一基因，并 在目标区间含有 $1212 \mathrm{bp}$ 的大片段缺失。Weng等 ${ }^{[15]}$ 对GW5 的核酸序列进行蛋白结构预测发现其具有一 个核定位信号和一个精氨酸富集结构域，并对 GW5 的序列分析表明其编码一种新的蛋白, 与任何已知 功能的蛋白都不具有同源性，而且酵母双杂交实验 发现GW5 与多聚遍在蛋白发生相互作用, 从而认为 GW5 调控水稻种子粒形是参与了泛素蛋白酶体途 径。Shomura等 ${ }^{[16]}$ 对 qSW5 的组织切片观察发现, 粒 宽的增大主要原因在于外稃大小的差异, 外稃增大 在于上表皮细胞数量增加。

4 水稻粒形性状在超高产育种中的应用研 究与展望

我国水稻单产水平在经历了矮化育种和杂交 
稻育种这两次大的飞跃后, 长期停滞不前, 为了打 破这种局面，实现水稻单产水平的第三次飞跃，20 世 纪 80 年代, 日本、韩国、IRRI(International Rice Research Institute)、中国相继提出并开展了理想株型与杂 种优势利用相结合的“水稻超高产育种研究” [7,61,62]。 作物育种的实践表明，突破性成就都依赖于特异种 质的发现及育种材料的构建 ${ }^{[63]}$ ，例如水稻矮秆基 因、水稻野败胞质以及光温敏特性材料的发掘利用, 使水稻产量都有了大幅度提高。水稻粒形是影响水 稻粒重(千粒重)的重要产量相关性状, 现在已克隆 了一批控制水稻粒形的重要基因 ${ }^{[15,16,44,47]}$ 以及与产 量性状相关的基因(表 2)，这不仅有助于揭示水稻产 量性状复杂的遗传机制, 而且为水稻的分子标记辅 助选择提供了理论依据和技术基础。

Fan等 ${ }^{[64]}$ 对 170 份水稻初级核心种质材料 ${ }^{[65]}$ 和 10 份国外水稻品种进行研究发现GS3 第 2 个外显子 中 C-A单碱基突变(SNP)与粒长性状高度关联, 由此 开发出 1 个功能性标记SF28, 可以应用于分子标记 辅助选择GS3 基因, 以改良稻米外观和产量。Song 等 ${ }^{[47]}$ 对 GW2 做了育种应用的产量品质两方面的深入 研究, 结果发现NIL(GW2)相比丰矮占 1(FAZ1), 虽然 主穗粒数减少 $29.9 \%$, 但单株谷粒产量提高 $19.7 \%$, 并且来自WY3 的 GW2 等位基因并不会影响FAZ1 的 株叶形态、籽粒灌浆以及稻米的蒸煮和食用品质。 Jiao等 ${ }^{[52]}$ 最近克隆了一个具有一因多效的IPA1 基因, 其表达能减少分菜, 增强植株抗倒伏能力以及增加 谷物产量, 从而具有塑造理想株型和提高产量的育 种潜力。Ashikari等 ${ }^{[50,66,67]}$ 通过发展遗传背景为 Koshihikari，而含有来自Habataki的控制谷粒数的等 位基因 $G n 1$ 和控制株高的等位基因 $S d 1$ 的NIL-Gn1 和NIL-Sd1, 利用杂交和篮选获得NIL-Gn1- Sd1, 该株 系相比Koshihikari能增加 $26 \%$ 的谷粒产量, 而使株 高降低 $18 \%$ 。Miura等 ${ }^{[53]}$ 利用Nipponbare和ST-12 杂 交分离群体在第 1 染色体上找到控制穗粒数 $G n 1 a^{[50]}$ 和在第 8 染色体上找到控制一次枝梗数的WFP, 通 过在 $\mathrm{BC}_{2} \mathrm{~F}_{2}$ 群体中篮选到含有 $G n 1 a$ 和 WFP两基因的 4 种不同组合株系, 比较不同株系间每穗一次枝梗 数和每穗粒数, 发现来自 ST-12 的 Gn1a和 WFP两基 因的聚合能增加 $40 \% \sim 50 \%$ 的穗粒数，从而提高水稻 产量。

虽然现在国内外科学家已成功克隆到控制水稻
粒形的重要基因 ${ }^{[15,16,44,47]}$, 以及与粒形性状相关的 基因(表 2)，从而知道控制水稻粒形的分子调控的可 能途径和机理, 例如某些跨膜蛋白能参与对生长因 子信号通路的调节控制来抑制器官正常生长发育, 或具有蛋白降解功能的泛素蛋白酶体途径可以通过 降解控制粒形的相关蛋白而实现对粒形的影响, 这 些功能的行使都基于编码功能蛋白的基因核酸序列 发生单碱基缺失、替换或大片段缺失，从而使功能 蛋白失活, 使水稻谷粒变长(变宽), 从而增加粒重, 但这些研究结果除了对控制水稻粒形的分子机理有 所启示外，其实际育种应用还比较难，例如有些基 因资源可能来源于籼稻和粳稻分化前的自然选择或 籼粳分化后的人为选择，以及某些基因功能的行使 需要不同遗传背景下的特异遗传因子 ${ }^{[55,64]}$, 以及如 何克服水稻粒形变大增加粒重所带来的稻米品质下 降问题, 以及水稻超高产育种中即要求谷物产量高 又要求其具有理想株型。所以水稻重要农艺性状的 遗传改良，除了利用单一基因进行遗传改良外，挖 掘具有一因多效的关键基因资源或采用基因聚合的途 径能更快和更有效的培育理想的超高产水稻品种。

\section{参考文献(References):}

[1] 龙彭年. 杂交水稻走向世界的现状与发展对策. 中国稻米, 2004, 11(5): 6-8. [DOI]

[2] Wang YH, Li JY. Branching in rice. Curr Opin Plant Biol, 2010, 14: 1-6. [DOI]

[3] Fitzgerald MA, McCouch SR, Hall RD. Not just a grain of rice: the quest for quality. Trends Plant Sci, 2009, 14(3): 133-139. [DOI]

[4] 吴绍洪, 李荣生. 中国耕地与未来 30 年食物需求、保障及 对策. 地理科学进展, 2002, 21(2): 121-129. [DOI]

[5] 赵明, 李建国, 张宾, 董志强, 王美云. 论作物高产挖潜的 补偿机制. 作物学报, 2006, 32(10): 1566-1573. [DOI]

[6] 程式华, 曹立勇, 庄杰云, 吴伟明. 关于超级稻品种培育 的资源和基因利用问题. 中国水稻科学，2009，23(3): 223-228. [DOI]

[7] 陈温福, 徐正进, 张文忠, 张龙步, 杨守仁. 水稻新株型创 造与超高产育种. 作物学报, 2001, 27(5): 665-672. [DOI]

[8] 熊振民, 孔繁林. 水稻大穗大粒型育种的研究. 江苏农业 科学, 1981, (4): 25-30.

[9] 徐正进, 陈温福, 马殿荣, 吕英娜, 周淑清, 刘丽霞. 稻谷 粒形与稻米主要品质性状的关系. 作物学报, 2004, 30(9): 894-900. [DOI]

[10] 莫惠栋. 谷类作物胚乳品质性状的遗传研究. 中国农业科 学, 1995, 28(2): 1-7. [DOI] 
[11] 徐建龙, 薛庆中, 罗利军, 黎志康. 水稻粒重及其相关性 状的遗传解析. 中国水稻科学, 2002, 16(1): 6-10. [DOI]

[12] 姚国新, 卢磊. 水稻粒重基因定位克隆研究. 安徽农业科 学, 2007, 35(27): 8468, 8478. [DOI]

[13] 莫惠栋. 我国稻米品质的改良. 中国农业科学, 1993, 26(4): 8-14. [DOI]

[14] 刘明伟, 刘勇, 王世全, 邓其明, 李平. 水稻显性小粒基因 $M i 3(t)$ 的遗传定位. 中国水稻科学, 2005, 19(6): 511-515. [DOI]

[15] Weng JF, Gu SH, Wan XY, Gao H, Guo T, Su N, Lei CL, Zhang X, Cheng ZJ, Guo XP, Wang JL, Jiang L, Zhai HQ, Wan JM. Isolation and initial characterization of GW5, a major QTL associated with rice grain width and weight. Cell Res, 2008, 18(12): 1199-1209. [DOI]

[16] Shomura A, Izawa T, Ebana K., Ebitani T, Kanegae H, Konishi S, Yano M. Deletion in a gene associated with grain size increased yields during rice domestication. Nat Genet, 2008, 40(8): 1023-1028. [DOI]

[17] 郭益全, 劉清. 大粒水稻之遺傳研究 II. 款粒性狀之遺傳. 中華農業研究, 1986, 35(4): 401-412. [DOI]

[18] 杨联松, 白一松, 陈多璞, 杨剑波, 丁超尘. 粳稻粒形遗传 初步研究. 杂交水稻, 2002, 17(6): 46-48. [DOI]

[19] 熊振民, 孔繁林. 水稻粒重的超亲遗传及其在育种中的应 用. 浙江农业大学学报(农业与生命科学版), 1982, 8(1): 17-25. [DOI]

[20] 石春海, 申宗坦. 早籼粒形的遗传和改良. 中国水稻科学, 1995, 9(1): 27-32. [DOI]

[21] 石春海, 申宗坦. 籼稻粒形及产量性状的加性相关和显性 相关分析. 作物学报, 1996, 22(1): 36-42. [DOI]

[22] 祈祖白, 李宝健, 杨文广, 吴秀峰. 水稻籽粒外观品质及 脂肪的遗传研究. 遗传学报, 1983, 10(6): 452-458. [DOI]

[23] 易小平, 陈芳远. 籼型杂交水稻品质性状的细胞质遗传效 应研究. I. 稻米外观品质及氨基酸含量分析. 广西农学院 学报, 1991, 10(1): 25-32. [DOI]

[24] 符福鸿, 王丰, 黄文剑, 彭惠普, 伍应运, 黄德娟. 杂交水 稻谷粒性状的遗传分析. 作物学报, 1994, 20(1): 39-45. [DOI]

[25] 石春海, 何慈信, 朱军, 陈建国. 籼稻稻米外观品质性状 的遗传主效应和环境互作效应分析. 中国水稻科学, 1999, 13(3): 179-182. [DOI]

[26] Zhu J. Analysis of conditional genetic effects and variance components in development genetics. Genetics, 1995, 141(4): 1633-1639. [DOI]

[27] 石春海, 吴建国, 蒋淑丽. 籼稻稻米粒长和粒宽性状的发 育遗传研究. 中国作物学会水稻产业分会成立大会暨首届 中国稻米论坛, 2003: 96-99. [DOI]

[28] 石春海, 吴平, 吴建国, 朱军, 焚龙江. 籼稻精米重量性状 的发育遗传分析. 浙江大学学报(农业与生命科学版), 2001, 27(5): 483-488. [DOI]

[29] Yano M, Sasaki T. Genetic and molecular dissection of quantitative traits in rice. Plant Mol Biol, 1997, 35(1-2): 145-153.[DOI]
[30] 庄杰云, 郑康乐. 水稻产量性状遗传机理及分子标记辅助 高产育种. 生物技术通报, 1998, (1): 1-9. [DOI]

[31] 严长杰, 顾铭洪. 高代回交QTL分析与水稻育种. 遗传, 2000, 22(6): 419-422. [DOI]

[32] 姜树坤, 徐正进, 陈温福. 水稻QTL图位克隆的特征分析. 遗传, 2008, 30(9): 1121-1126. [DOI]

[33] Paterson AH, Lander ES, Hewitt JD, Peterson S, Lincoln SE, Tanksley SD. Resolution of quantitative traits into Mendelian factors by using a complete linkage map of restriction fragment length polymorphisms. Nature, 1988, 335(6192): 721-726. [DOI]

[34] 毛传澡, 程式华. 水稻农艺性状QTL定位精确性及其影响 因素的分析. 农业生物技术学报，1999，7(4)：386-394. [DOI]

[35] Xing Z, Tan F, Hua P, Sun L, Xu G, Zhang Q. Characterization of the main effects, epistatic effects and their environmental interactions of QTLs on the genetic basis of yield traits in rice. Theor Appl Genet, 2002, 105(2-3): 248-257. [DOI]

[36] Tan YF, Li JX, Yu SB, Xing YZ, Xu CG, Zhang QF. The three important traits for cooking and eating quality of rice grains are controlled by a single locus in an elite rice hybrid, Shanyou 63. Theor Appl Genet, 1999, 99(3-4): 642-648. [DOI]

[37] Tsunematsu H, Yoshimura A, Harushima Y, Nagamura Y, Kurata N, Yano M, Sasaki T, Iwata N. RFLP framework map using recombinant inbred lines in rice. Breed Sci, 1996, 46(3): 279-284. [DOI]

[38] Li JM, Xiao JH, Grandillo S, Jiang LY, Wan YZ, Deng QY, Yuan LP, McCouch SR. QTL detection for rice grain quality traits using an interspecific backcross population derived from cultivated Asian (O. sativa L.) and African (O. glaberrima S.) rice. Genome, 2004, 47(4): 697-704. [DOI]

[39] Yoshida S, Ikegami M, Kuze J, Sawada K, Hashimoto Z, Ishii T, Nakamura C, Kamijima O. QTL analysis for plant and grain characters of sake-brewing rice using a doubled haploid population. Breed Sci, 2002, 52(4): 309-317. [DOI]

[40] Aluko G, Martinez C, Tohme J, Castano C, Bergman C, Oard JH. QTL mapping of grain quality traits from the interspecific cross Oryza sativa $\times$ O. glaberrima. Theor Appl Genet, 2004, 109(3): 630-639. [DOI]

[41] Ramalingam J, Vera Cruz CM, Kukreja K, Chittoor JM, Wu JL, Lee SW, Baraoidan M, George ML, Cohen MB, Hulbert $\mathrm{SH}$, Leach JE, Leung H. Candidate defense genes from rice, barley, and maize and their association with qualitative and quantitative resistance in rice. Mol Plant-Microbe Interact, 2003, 16(1): 14-24. [DOI]

[42] Redoña ED, Mackill DJ. Quantitative trait locus analysis for rice panicle and grain characteristics. Theor Appl Genet, 1998, 96(6-7): 957-963. [DOI]

[43] Cai HW, Morishima H. QTL clusters reflect character associations in wild and cultivated rice. Theor Appl Genet, 2002, 104(8): 1217-1228. [DOI] 
[44] Fan CC, Xing YZ, Mao HL, Lu TT, Han B, Xu CG, Li XH, Zhang QF. GS3, a major QTL for grain length and weight and minor QTL for grain width and thickness in rice, encodes a putative transmembrane protein. Theor Appl Genet, 2006, 112(6): 1164-1171. [DOI]

[45] 陈志军, 汤在祥, 宋雯, 徐辰武. 基于遗传位置的水稻与 玉米重要农艺性状QTL比较研究. 中国水稻科学, 2009, 23(3): 229-236. [DOI]

[46] Goff SA, Ricke D, Lan TH, Presting G, Wang R, Dunn M, Glazebrook J, Sessions A, Oeller P, Varma H, Hadley D, Hutchison D, Martin C, Katagiri F, Lange BM, Moughamer T, Xia Y, Budworth P, Zhong J, Miguel T, Paszkowski U, Zhang S, Colbert M, Sun WL, Chen L, Cooper B, Park S, Wood TC, Mao L, Quail P, Wing R, Dean R, Yu Y, Zharkikh A, Shen R, Sahasrabudhe S, Thomas A, Cannings R, Gutin A, Pruss D, Reid J, Tavtigian S, Mitchell J, Eldredge G, Scholl T, Miller RM, Bhatnagar S, Adey N, Rubano T, Tusneem N, Robinson R, Feldhaus J, Macalma T, Oliphant A, Briggs S. A draft sequence of the rice genome (Oryza sativa L. ssp. japonica). Science, 2002, 296(5565): 92-100. [DOI]

[47] Song XJ, Huang W, Shi M, Zhu MZ, Lin HX. A QTL for rice grain width and weight encodes a previously unknown RING-type E3 ubiquitin ligase. Nat Genet, 2007, 39(5): 623-630. [DOI]

[48] Wang E, Wang J, Zhu X, Hao W, Wang L, Li Q, Zhang L, He W, Lu B, Lin H, Ma H, Zhang G, He Z. Control of rice grain-filling and yield by a gene with a potential signature of domestication. Nat Genet, 2008, 40(11): 1370 - 1374. [DOI]

[49] Xue WY, Xing YZ, Weng XY, Zhao Y, Tang WJ, Wang L, Zhou HJ, Yu SB, Xu CG, Li XH, Zhang QF. Natural variation in Ghd7 is an important regulator of heading date and yield potential in rice. Nat Genet, 2008, 40(6): 761-767. [DOI]

[50] Ashikari M, Sakakibara H, Lin SY, Yamamoto T, Takashi T, Nishimura A, Angeles ER, Qian Q, Kitano H, Matsuoka M. Cytokinin oxidase regulates rice grain production. Science, 2005, 309(5735): 741-745. [DOI]

[51] Huang XZ, Qian Q, Liu ZB, Sun HY, He SY, Luo D, Xia GM, Chu CC, Li JY, Fu XD. Natural variation at the DEP1 locus enhances grain yield in rice. Nat Genet, 2009, 41(4): 494-497. [DOI]

[52] Jiao YQ, Wang YH, Xue DW, Wang J, Yan MX, Liu GF, Dong GJ, Zeng DL, Lu ZF, Zhu XD, Qian Q, Li JY. Regulation of OsSPL14 by OsmiR156 defines ideal plant architecture in rice. Nat Genet, 2010, 42(6): 541-544. [DOI]

[53] Miura K, Ikeda M, Matsubara A, Song XJ, Ito M, Asano K, Matsuoka M, Kitano H, Ashikari M. OsSPL14 promotes panicle branching and higher grain productivity in rice. Nat Genet, 2010, 42(6): 545-549. [DOI]

[54] Tan YF, Xing YZ, Li JX, Yu SB, Xu CG, Zhang QF. Genetic bases of appearance quality of rice grains in Shanyou 63, an elite rice hybrid. Theor Appl Genet, 2000, 101(5-6): 823-829. [DOI]

[55] Takano-Kai N, Jiang H, Kubo T, Sweeney M, Matsumoto T, Kanamori H, Padhukasahasram B, Bustamante C, Yoshimura A, Doi K, McCouch S. Evolutionary history of GS3, a gene conferring grain length in rice. Genetics, 2009, 182(4): 1323-1334. [DOI]

[56] Itoh J, Nonomura K, Ikeda K, Yamaki S, Inukai Y, Yamagishi H, Kitano H, Nagato Y. Rice plant development: from zygote to spikelet. Pant Cell Physiol, 2005, 46(1): 23-47. [DOI]

[57] Van Vlijmen HWT, Gupta A, Narasimhan LS, Singh J. A novel database of disulfide patterns and its application to the discovery of distantly related homologs. J Mol Biol, 2004, 335(4): 1083-1092. [DOI]

[58] Zhang JL, Huang Y, Qiu LY, Nickel J, Sebald W. von Willebrand factor type $\mathrm{C}$ domain-containing proteins regulate bone morphogenetic protein signaling through different recognition mechanisms. J Biol Chem, 2007, 282(27): 20002-20014. [DOI]

[59] Garcia Abreu J, Coffinier C, Larraín J, Oelgeschläger M, De Robertis EM. Chordin-like CR domains and the regulation of evolutionary conserved extracellular signaling systems. Gene, 2002, 287(1-2): 39-47.

[60] O’Leary JM, Hamilton JM, Deane CM, Valeyev NV, Sandell LJ, Downing AK. Solution structure and dynamics of a prototypical Chordin-rich repeat (von Willebrand Factor type C module) from collagen IIA. J Biol Chem, 2004, 279(51): 53857-53866. [DOI]

[61] 邹江石, 吕川根. 水稻超高产育种的实践与思考. 作物学 报, 2005, 31(2): 254-258. [DOI]

[62] 冯荣坤. 超高产水稻育种的研究进展. 湖南农业科学, 2006, (3): 19-22. [DOI]

[63] 程式华. 杂交水稻育种材料和方法研究的现状及发展趋势. 中国水稻科学, 2000, 14(3): 165-169. [DOI]

[64] Fan CC, Yu SB, Wang CG, Xing YZ. A causal C-A mutation in the second exon of GS3 highly associated with rice grain length and validated as a functional marker. Theor Appl Genet, 2009, 118(3): 465-472. [DOI]

[65] 李自超, 张洪亮, 曹永生, 表宗恩, 魏兴华, 汤圣祥, 余萍, 王象坤. 中国地方稻种资源初级核心种质取样策略研究. 作物学报, 2003, 29(1): 20-24. [DOI]

[66] Ashikari M, Matsuoka M. Identification, isolation and pyramiding of quantitative trait loci for rice breeding. Trends Plant Sci, 2006, 11(7): 344-350. [DOI]

[67] Ashikari M, Sasaki A, Ueguchi Tanaka M, Itoh H, Nishimura A, Datta S, Ishiyama K, Saito T, Kobayashi M, Khush GS, Kitano H, Matsuoka M. Loss of function of a rice gibberellin biosynthetic gene, GA20 oxidase (GA20ox-2), lead to the rice "Green Revolution". Breed Sci, 2002, 52(2): 143-150. [DOI] 